\title{
EXTENSION OF AN EXISTENCE THEOREM FOR A NON-SELF-ADJOINT LINEAR SYSTEM.
}

BY PROFESSOR H. J. ETTLINGER.

(Read before the American Mathematical Society December 31, 1919.)

In a recent paper* the writer established the existence of at least one real characteristic number for the non-self-adjoint system

(2) $\quad U_{i}=A_{i 1} u(a)-A_{i 2} K(a) u_{x}(a)$

$$
-A_{i 3} u(b)+A_{i 4} K(b) u_{x}(b)=0 \quad(i=1,2),
$$

satisfying the following conditions I, II, III, IV, V, and either VI A or VI B:

I. $K(x, \lambda)$ and $G(x, \lambda)$ are continuous, real functions of $x$ in $a \leqq x \leqq b$ and for all real values of $\lambda$ in the interval

$$
\Lambda\left(\Lambda_{1}<\lambda<\Lambda_{2}\right) .
$$

II. $K(x, \lambda)$ is positive everywhere in $(a, b), \Lambda$.

III. The sets of real constants $A_{1 j}$ and $A_{2 j}$ are not proportional.

IV. For each value of $x$ in $(a, b), K$ and $G$ do not increase as $\lambda$ increases.

V. $\lim _{\lambda=\Lambda_{1}}-\frac{\min G}{\min K}=-\infty, \lim _{\lambda=\Lambda_{2}}-\frac{\max G}{\max K}=+\infty$.

VI A. $\dagger D_{12} \cdot D_{34}=0$, together with either

$$
D_{24}{ }^{2}+D_{14}{ }^{2} \neq 0, \quad D_{14} \geqq 0, \quad D_{24} \geqq 0,
$$

or

(b) $D_{24}{ }^{2}+D_{14}{ }^{2}=0, \quad D_{13}{ }^{2}+D_{23}{ }^{2} \neq 0, \quad D_{13} \leqq 0, \quad D_{23} \leqq 0$.

VI B. $D_{12} \cdot D_{34} \neq 0$, together with either

$$
\text { (a) } D_{24}>0 \text { or (b) } D_{24}=0, D_{14}>0 \text {. }
$$

* Existence theorem for the non-self-adjoint linear system of the second order, AnNals of Mathematics, vol. 25 (1920), pp. 278-290.

$$
\dagger D_{i j}=\left|\begin{array}{ll}
A_{1 i} & A_{1 j} \\
A_{2 i} & A_{2 j}
\end{array}\right| \text {. }
$$


It is the purpose of this note to extend this theorem to the non-self-adjoint system

$$
\frac{d}{d x}\left[K(x, \lambda) \frac{d u}{d x}\right]-G(x, \lambda) u=0,
$$

(3) $\quad U_{i}=A_{i 1}(\lambda) u(a)-A_{i 2}(\lambda) K(a) u_{x}(a)-A_{i 3}(\lambda) u(b)$

$$
+A_{i 4}(\lambda) K(b) u_{x}(b)=0 \quad(i=1,2),
$$

satisfying conditions I, II, IV, V, and the following new conditions: III', either $\mathrm{VI}^{\prime} \mathrm{A}$ or $\mathrm{VI}^{\prime} \mathrm{B}$, either VII A or VII B or VII C or VII D or VII E, and VIII.

III'. $A_{1 j}(\lambda)$ and $A_{2 j}(\lambda)$ are two independent sets of functions continuous in $\lambda$ throughout $\Lambda$.

VI $A . \quad D_{12}(\lambda) \equiv 0, D_{34}(\lambda) \neq 0$, or $D_{12}(\lambda) \neq 0, D_{34}(\lambda) \equiv 0$ together with either

(a) $D_{24}{ }^{2}(\lambda)+D_{14}{ }^{2}(\lambda) \neq 0, \quad D_{14}\left(\Lambda_{1}+\epsilon\right) \geqq 0, \quad D_{24}\left(\Lambda_{1}+\epsilon\right) \geqq 0$, or

(b) $\quad D_{24}{ }^{2}(\lambda)+D_{14}{ }^{2}(\lambda) \equiv 0, \quad D_{13}{ }^{2}(\lambda)+D_{23}{ }^{2}(\lambda) \neq 0$,

$$
D_{13}\left(\Lambda_{1}+\epsilon\right) \leqq 0, \quad D_{23}\left(\Lambda_{1}+\epsilon\right) \leqq 0 .
$$

VI' B. $D_{12}(\lambda) \cdot D_{34}(\lambda) \neq 0$, together with either

$$
D_{24}\left(\Lambda_{1}+\epsilon\right)>0 \text {, }
$$

or

(b)

VII $A$.

$$
D_{24}(\lambda) \equiv 0, \quad D_{14}\left(\Lambda_{1}+\epsilon\right)>0 .
$$

$$
D_{24}(\lambda) \neq 0, \quad \Delta\left[\frac{D_{14}(\lambda)}{D_{24}(\lambda)}\right]^{*} \leqq 0, \quad \Delta\left[\frac{D_{23}(\lambda)}{D_{24}(\lambda)}\right] \leqq 0 .
$$

VII $B$.

$$
D_{14}(\lambda) \neq 0, \quad \Delta\left[\frac{D_{14}(\lambda)}{D_{24}(\lambda)}\right] \leqq 0,
$$

together with either $D_{24}(\lambda) \equiv 0$, or

VII $C$.

$$
D_{24}(\lambda) \neq 0 \text { and } \Delta\left[\frac{D_{14}(\lambda)}{D_{24}(\lambda)}\right] \leqq 0 .
$$

$$
D_{23}(\lambda) \neq 0, \quad \Delta\left[\frac{D_{13}(\lambda)}{D_{23}(\lambda)}\right] \leqq 0,
$$

${ }^{*} \Delta \phi(\lambda)=\phi(\lambda+\Delta \lambda)-\phi(\lambda)$ for $\Delta \lambda>0$. 
together with either $D_{14}(\lambda) \equiv 0$, or

VII $D$.

$$
D_{14}(\lambda) \neq 0 \text { and } \Delta\left[\frac{D_{13}(\lambda)}{D_{14}(\lambda)}\right] \leqq 0 .
$$

$$
D_{23}(\lambda) \neq 0 \text { and } \Delta\left[\frac{D_{13}(\lambda)}{D_{23}(\lambda)}\right] \leqq 0,
$$

together with either $D_{24}(\lambda) \equiv 0$, or

$$
D_{24}(\lambda) \neq 0 \text { and } \Delta\left[\frac{D_{23}(\lambda)}{D_{24}(\lambda)}\right] \leqq 0 .
$$

VII $E$.* Either $D_{23}(\lambda) \equiv 0$, or

$$
D_{23}(\lambda) \neq 0 \text { and } \Delta\left[\frac{D_{13}(\lambda)}{D_{23}(\lambda)}\right] \leqq 0,
$$

together with either $D_{24}(\lambda) \equiv 0$, or

$$
D_{24}(\lambda) \neq 0 \text { and } \Delta\left[\frac{D_{14}(\lambda)}{D_{24}(\lambda)}\right] \leqq 0 .
$$

VIII. $\Delta D_{13} \cdot \Delta D_{24}-\Delta D_{23} \cdot \Delta D_{14} \leqq 0$.

The types of (3) which we shall consider are the following:

Type I. (a) $D_{12}(\lambda) \equiv 0, \quad D_{34}(\lambda) \neq 0$,

$$
\text { (b) } D_{12}(\lambda) \neq 0, \quad D_{34}(\lambda) \equiv 0 \text {. }
$$

Type II. $\quad D_{12}(\lambda) \cdot D_{34}(\lambda)>0$.

Type III. $D_{12}(\lambda) \cdot D_{34}(\lambda)<0$.

Then the various sets of non-self-adjoint conditions $\dagger$ are

$$
\begin{aligned}
& \text { A. }\left(\begin{array}{cccc}
D_{12}(\lambda) & 0 & D_{32}(\lambda) & D_{42}(\lambda) \\
D_{14}(\lambda) & D_{24}(\lambda) & 0 & 0
\end{array}\right) \\
& \text { B. }\left(\begin{array}{cccc}
0 & D_{12}(\lambda) & D_{13}(\lambda) & D_{24}(\lambda) \\
D_{14}(\lambda) & D_{24}(\lambda) & 0 & 0
\end{array}\right) \\
& \text { C. }\left(\begin{array}{cccc}
0 & D_{12}(\lambda) & D_{13}(\lambda) & D_{14}(\lambda) \\
D_{13}(\lambda) & D_{23}(\lambda) & 0 & 0
\end{array}\right) \\
& \text { D. }\left(\begin{array}{cccc}
D_{12}(\lambda) & 0 & D_{32}(\lambda) & D_{42}(\lambda) \\
D_{13}(\lambda) & D_{23}(\lambda) & 0 & 0
\end{array}\right) \\
& \text { E. }\left(\begin{array}{llll}
D_{13}(\lambda) & D_{23}(\lambda) & 0 & D_{43}(\lambda) \\
D_{14}(\lambda) & D_{24}(\lambda) & D_{34}(\lambda) & 0
\end{array}\right) .
\end{aligned}
$$

* Evidently the alternative conditions VII hold for the corresponding set of conditions enumerated later.

$\dagger$ See Annals of Mathematics, loc. cit. 
These conditions ensure the validity of the theorems* used in the proof of the existence theorem stated above, and the proof follows exactly as in the original theorem.

UNIVERSITY OF TEXAs,

July 30, 1920.

\section{ON THE CAUCHY-GOURSAT THEOREM.}

BY R. L. BORGER.

(Read before the American Mathematical Society December 30,1919.)

In order to prove his integral theorem, viz: $J_{c} f(z) d z=0$, Cauchy found it necessary to assume not only that the derivative $f^{\prime}(z)$ existed but also that it was continuous. Later, proofs were given by Goursat and by Moore $\dagger$ in which the mere existence of $f^{\prime}(z)$ was shown to be sufficient for the truth of the theorem. These were based upon the analysis of the complex variable.

From the standpoint of the real variable many interesting investigations have developed around the Cauchy-Goursat theorem. They have depended upon Green's theorem. Porter, $\ddagger$ using the Riemann integral, proved that with proper restrictions upon the component functions, $U$ and $V$, of the complex function, Green's theorem was true, and hence that Cauchy's integral theorem was also true, even when the derivative $f^{\prime}(z)$ did not exist. Montel, $\S$ by means of the Lebesgue integral, proved Green's theorem under the hypothesis that $U_{x}, V_{y}$, exist, are bounded, and satisfy the equation

$$
U_{x}=V_{y},
$$

except at most in a set of measure zero. He was then able to prove the integral theorem, and the existence of the derivative $f^{\prime}(z)$ for a function of the complex variable $f(z)$ in the closed region considered. The existence, then, of the deriva-

* Cf. Existence theorems for the general, real, self-adjoint linear system of the second order, Transactions Amer. Math. Soc., vol. 19 (1918), p. 94.

† Transactions Amer. Math. Society, vol. 1 (1900).

$\ddagger$ Annals of Mathematics, (2), vol. 7 (1905-6).

$\S$ Annales Scientifiques de l'Ecole normale Supérieure, (3), vol. 24 (1907). 